\title{
TRANSITION TO TURBULENCE IN STARTING PIPE FLOWS
}

\author{
TOSHIRO MARUYAMA, YOSHIEI KATO \\ AND TOKURO MIZUSHINA \\ Department of Chemical Engineering, Kyoto University, \\ Kyoto 606
}

\begin{abstract}
An experimental investigation was performed to study the transition to turbulence in pipe flow started impulsively with a supercritical Reynolds number. The results show that a turbulentnon-turbulent interface propagates downstream by consuming the non-turbulent region where laminar boundary-layer flow is developing with time. For the startup of a completely quieted fluid, the propagation velocity is equal to the maximum velocity of the steady-state turbulent flow. For restarting the fluid flow after a brief shutoff, however, the propagation velocity is larger than the maximum velocity, and the difference increases with shorter quieting times. In the process of interface propagation, a wave of very low frequency appears ahead of the interface centring around the radial position where the smallest change in velocity is observed at the instant of propagation.

A study of the mechanism of interface propagation shows that the final break of the abovementioned wave continuously triggers downstream propagation of the turbulent-flow region. In addition, random jumping of the interface in turbulence-decaying flow is concluded to be the main cause for the increase in propagation velocity for short quieting times.
\end{abstract}

\section{Introduction}

When pipe flow is started impulsively with a supercritical Reynolds number, there is an abrupt transition to turbulence during the flow transient. Since the time to reach turbulence from startup is relatively long, it is important to know this time for the dynamic control of pipe flow systems.

The transition to turbulence in unsteady flow is also interesting in connection with studies of the mechanism of laminar-turbulent transition in steady pipe flow. Recent studies ${ }^{2,5}$ of the transition in steady pipe flow have fairly well established that in fully developed laminar pipe flow it is triggered by unstable disturbances in the boundary layer in the entrance region of the pipe and that the turbulentflow region propagates downstream with a constant velocity independent of wall condition.

In a set of interesting experiments by Lindgren ${ }^{2)}$, he has suggested, on the basis of visual studies, the following splitting process of the turbulent slug at supercritical Reynolds numbers. The splitting process is due to spike formation within the disturbed flow of the pointed slug front with subsequent formation of new turbulent spots developing into the slug ahead of the original one, forming turbulent streaks which elongate as they propagate downstream. As

Received December 23. 1977. Correspondence concerning this article should be addressed to T. Maruyama. Y. Kato is now with Kawasaki Steel Corporation, Chiba 280. the Reynolds number increases, the turbulence appears more and more homogeneous and the elongation of the streaks appear to develop more continuously. Recently, Wygnanski and Champagne ${ }^{5}$ have suggested that a large eddy structure exists in the non-turbulent region ahead of the turbulent interface, but their measurements showed negligibly small velocity fluctuations in that region. Therefore, we need further experimental studies of the structure of the interface to determine the mechanism of interface propagation. Unfortunately, random occurrences of the transition in steady pipe flow prevent the use of an ensembleaveraging procedure in this type of experiment. Thus measurements of the transition to turbulence in starting flow are better because, regardless of inlet conditions it is very easy to regulate the occurrence of the transition by restrating the flow after shutting it down for a constant quieting time. Furthermore, the effects of decaying turbulence on the transition can be studied by varying the quieting time.

This paper sets out some details on both the time and mechanism of the transition to turbulence in transient flow after startup. Particular emphasis is placed on details of propagation of the turbulent-non-turbulent interface in connection with that in steady pipe flow.

\section{Experiments}

A schematic of the flow system is shown in Fig. 1. It is similar to the one used previously ${ }^{3,4)}$, except that the present system was equipped with an orifice plate 
at the inlet of the forerunning section to generate deliberate disturbances. Both impulsive startup and shutdown of the flow rate were made by quickly opening and closing a solenoid valve (Nakakita NS611NA-10) which required $0.09 \mathrm{sec}$ for opening and $0.07 \mathrm{sec}$ for closing.

A test section was located $60-80$ diameters downstream from the orifice plate along a single straight pipe $(5.1 \mathrm{~cm}$ inside diameter). In the test section both instantaneous velocity and wall shear stress were measured by an electrochemical method. A description of the velocity measurements and data processing appeared in an earlier paper $^{3)}$. To measure the wall shear stress, small platinum-wire electrodes $(0.01 \mathrm{~cm}$ diameter) were imbedded in the wall: six electrodes in the longitudinal direction and five in the circumferential direction. The electrical signals due to wall shear stress were simultaneously recorded on a chart of an electromagnetic oscillograph (Yokogawa Model 2924).

The working fluid was an aqueous solution ( $\rho=$ $1.045 \mathrm{~g} / \mathrm{cm}^{3}, \mu=0.01 \mathrm{~g} / \mathrm{cm} \cdot \mathrm{sec}$ at $25^{\circ} \mathrm{C}$ ) of 0.01 molar $\mathrm{K}_{4} \mathrm{Fe}(\mathrm{CN})_{6}$ and $\mathrm{K}_{3} \mathrm{Fe}(\mathrm{CN})_{6}$ with $1 \mathrm{~N} \mathrm{KOH}$ as a supporting electrolyte.

\section{Results and Discussion}

\section{1 Velocity of interface propagation}

An example of the wall shear stress measurements is shown in Fig. 2. The figure describes an experimental run with 'quieting time' $t_{q}$, the elapsed time after the previous experimental run, and 'propagation time' $t_{c}$, the time from the start of flow till the onset of turbulence. In the figure, the wall shear stress increases rapidly at the start of flow, decreases slowly, then suddenly increases with the onset of turbulence and indicates the usual following turbulent fluctuations. Simultaneous measurements at points along the test section showed that the propagation time increased with increasing distance $x$ (see Fig. 1) and that it was clearly distinguishable. That is, the transition to turbulence propagated downstream with a sharp interface. A conceptual sketch in Fig. 3 indicates that the transition to turbulence is similar to the propagation of turbulent slug in steady pipe flow with the exception that the non-turbulent starting-flow velocity profile is different. In this flow, the laminar boundary layer is developing with time whereas in steady pipe flow the velocity profile is that of fully developed Hagen-Poiseuille flow.

The preliminary study without the orifice plate showed that the transition was started by some obstacle, such as valves and elbows, immediately after the start of flow; the starting point changed with Reynolds number, because of the complex configuration of the obstacles. Therefore the starting point of

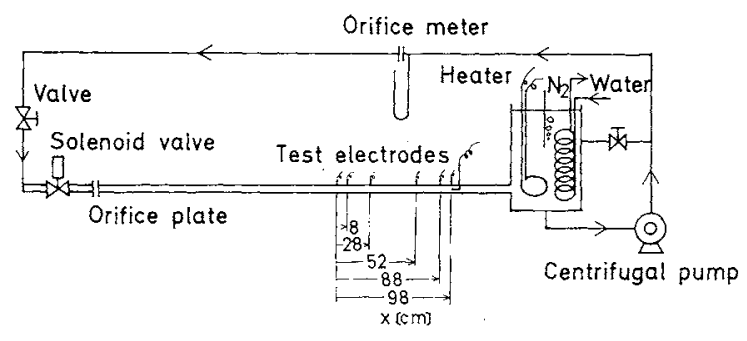

Fig. 1 Experimental apparatus

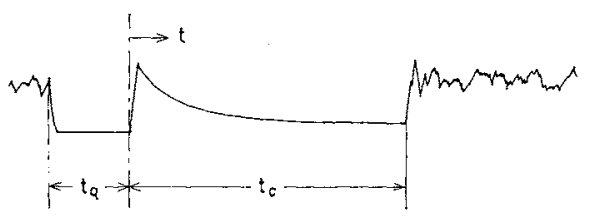

Fig. 2 Example of time variation of wall shear stress

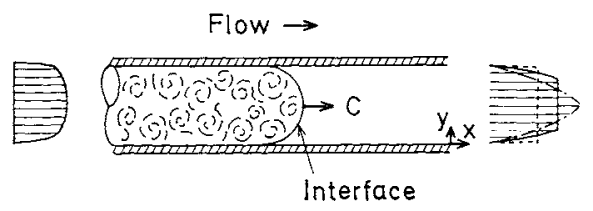

Fig. 3 Conceptual sketch of transition

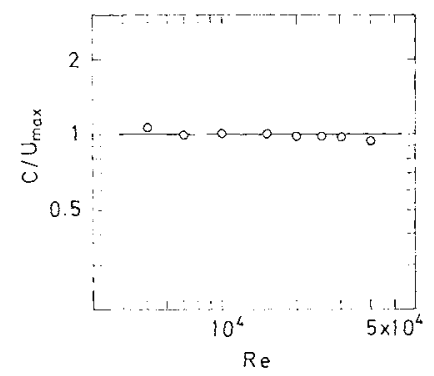

Fig. 4 Propagation velocity as a function of Reynolds number $\left(t_{q}=12 \mathrm{sec}\right)$

the transition studied was fixed by inserting the orifice plate in the inlet region of the straight test pipe. That the transition started at the orifice plate could be deduced from a clear decrease in the propagation times when an orifice plate having a small-enough opening was inserted.

The velocity $C$ of interface propagation was measured for various values of quieting time and Reynolds number by timing the interface between two wall shear stress meters. As a result, it was found that for long quieting times the interface propagated at constant velocity independent of quieting time and that propagation velocity increased with increasing Reynolds number. As shown in Fig. 4, propagation velocity $C$ is nearly equal to $U_{\mathrm{max}}$, the maximum velocity of the steady-state turbulent flow created by the transition to turbulence.

For short quieting times, however, it was found that 


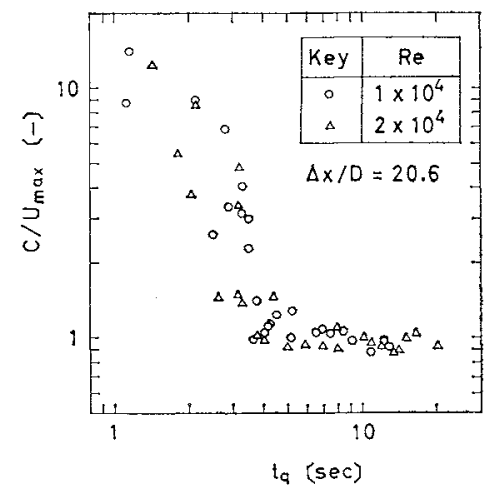

Fig. 5 Propagation velocity as a function of quieting time

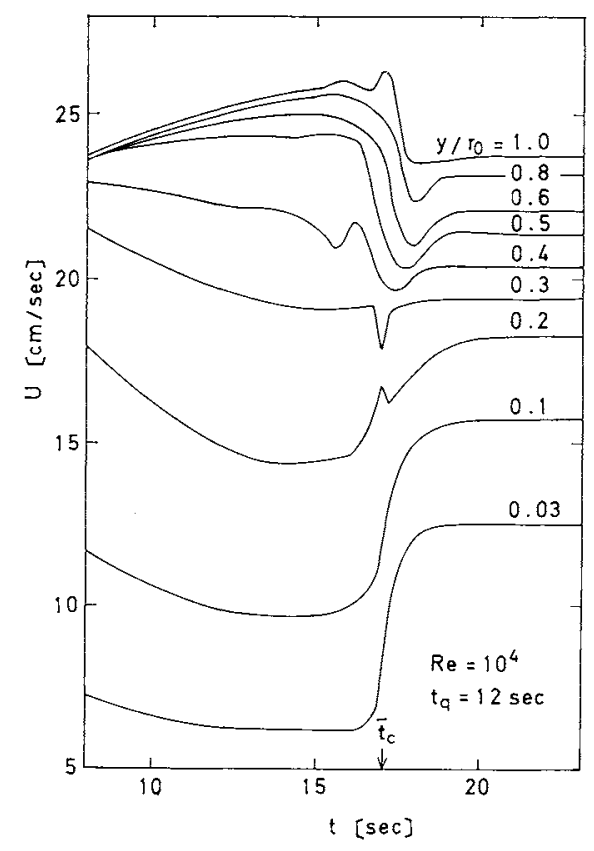

Fig. 6 Variation of ensemble-averaged velocities at various radial positions

propagation velocity was a function of quieting time. As seen in Fig. 5, propagation velocity became much larger than $U_{\max }$ for decreasing quieting times $t_{q}$ under about $4 \mathrm{sec}$. In addition, it varied as a function of longitudinal separation distance of electrode; this will be discussed in section 2.3. An inspection of the time charts of wall shear stress showed that acceleration of propagation velocity was associated with decaying turbulence. The decaying-turbulence effect was discernible in non-turbulent flow before the transition to turbulence for short quieting times.

On the basis of these facts, the mechanism of propagation is discussed firstly for turbulence-decayed flow and secondly for turbulence-decaying flow.

\section{2 Propagation in turbulence-decayed flow}

In this case the turbulence in the rest fluid has sufficiently decayed by startup of the next flow so that propagation velocity of the interface is independent

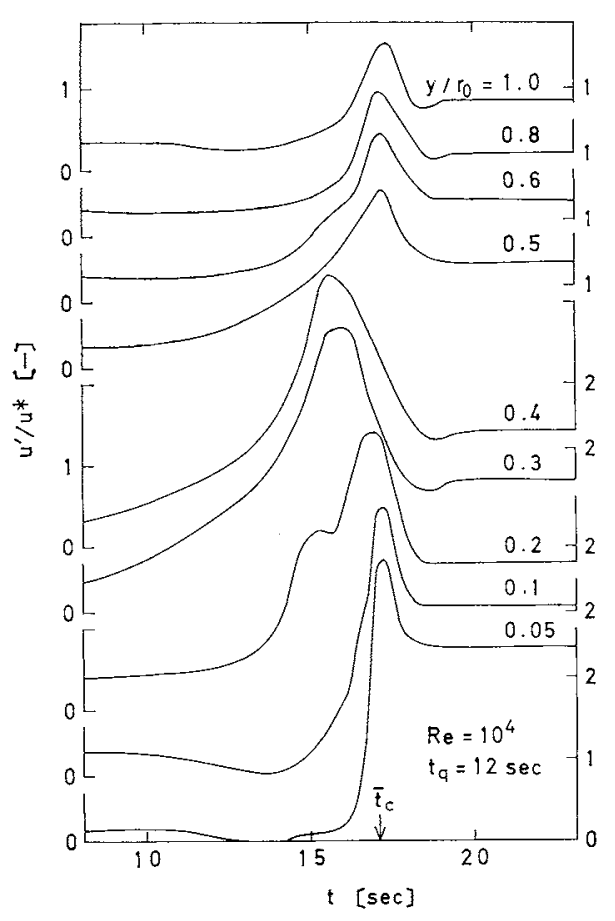

Fig. 7 Variation of intensity of $u$-fluctuations at various radial positions

of quieting time.

The variations in ensemble-averaged velocity at various radial positions are shown in Fig. 6 for $R e=$ $10^{4}, t_{q}=12 \mathrm{sec}$ and mean propagation time $\overline{t_{C}}=17 \mathrm{sec}$. At the propagation time, the velocities rapidly adjust to their new steady-state values, with velocities in the region $y / r_{0} \leq 0.2$ increasing and those in the region $y / r_{0} \geq 0.4$ decreasing. It can be noted that there appear some 'spikes' for $0.2 \leq y / r_{0} \leq 0.4$, where the velocity changes are smaller than those in the other regions.

The intensity of $u$-fluctuations are expressed as a dimensionless value, $u^{\prime} / u^{*}$ in Fig. 7 , where $u^{*}$ is the friction velocity in steady-state turbulent flow. The $u^{\prime} / u^{*}$ values rapidly approach their steady-state values at $t \simeq \overline{t_{\sigma}}$, showing a maximum just before the propagation of the interface. The maximum and the lead up to the maximum, however, depends on radial position.

At the position where the average-velocity change during propagation is minimum $\left(y / r_{0}=0.3\right.$ at $t \simeq \bar{t}_{C}^{-}$; see Fig. 6), the $u^{\prime} / u^{*}$ increase starts smoothly at $t \simeq 8 \mathrm{sec}$ and increases with time. On the other hand, at both $y / r_{0}=0.05$ and 1.0 , very rapid increases in $u^{\prime} / u^{*}$ are preceded by minimum values at $t \simeq 13.5 \mathrm{sec}$. These features are very similar to those observed by Klebanoff et al. ${ }^{1)}$ for wave growth at the spanwise position in transitional boundary-layer flow with $y / r_{0}=0.3$ corresponding to their 'peak' and both the wall and the tube axis to their 'valley'. 

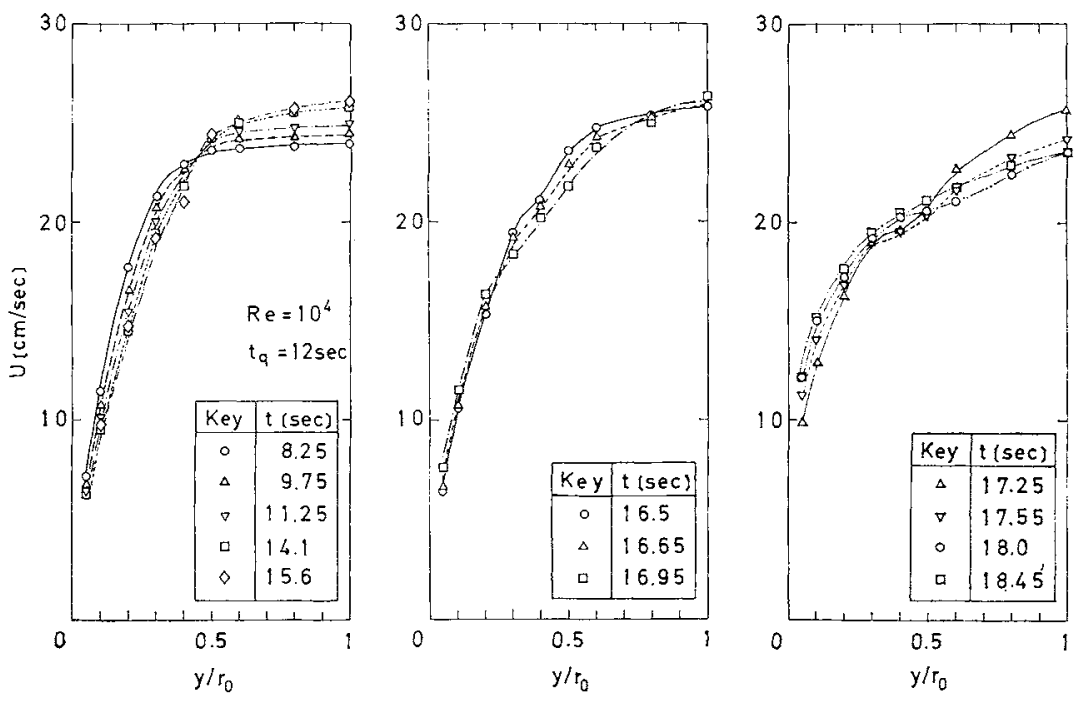

Fig. 8 Profiles of ensemble-averaged velocities
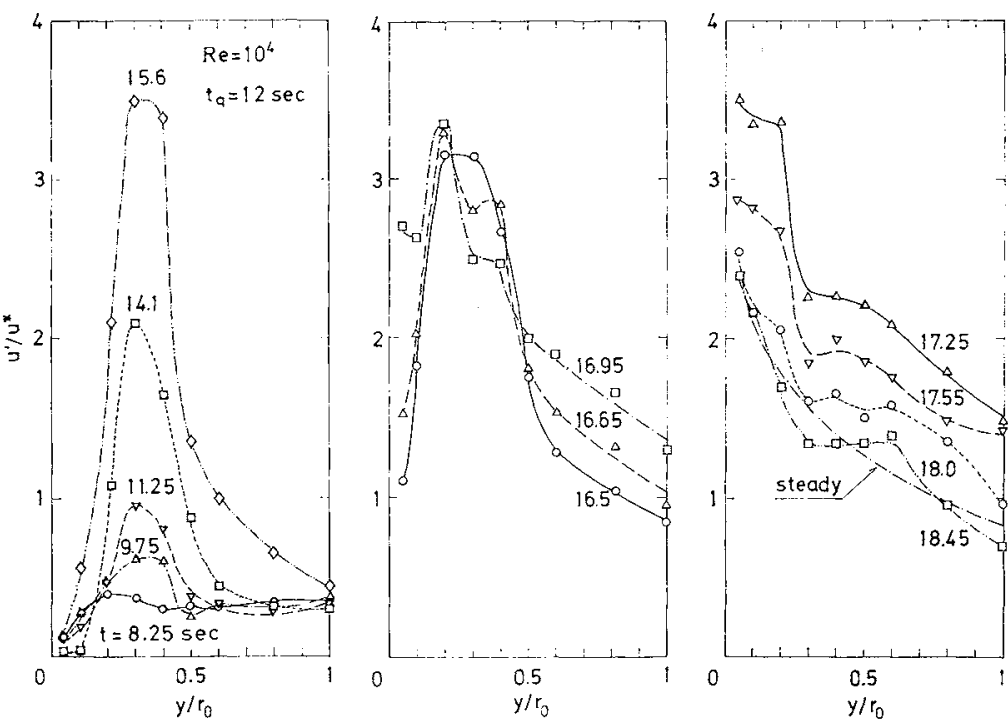

Fig. 9 Profiles of intensity of $u$-fluctuations

The variations in velocity in Fig. 6 are replotted in the form of velocity profiles in Fig. 8. A change before the propagation $(t \leq 16)$ suggests that the velocity profile would evolve from that of laminar boundarylayer flows to the parabolic profile, if the measuring position had been further downstream than in this study. During the propagation $(16 \leqq t \leqslant 17)$ the velocity profile develops an inflection point.

The corresponding profiles of the intensity of $u$ fluctuation are shown in Fig. 9. The development of the peak centring around the radial position $\left(y / r_{0}=\right.$ $0.3)$ is rather prominent $(t \leq 16)$. With the passing of the interface $(16 \leq t \leq 17)$, the peak rapidly disappears and then a dip occurs at this position. A comparison of the intensity profiles with the velocity profiles shows that the development of the peak does not affect the velocity profile of the laminar boundary layer. These changes in both velocity and its fluctuation are also similar to those observed in the transi- tional boundary layer in steady flow ${ }^{11}$, and suggest that the $u$-fluctuation amplifies according to the socalled 'Tollmien-Schlichting wave' of linear instability theory.

The energy spectra of the $u$-fluctuations at $y / r_{0}=0.3$ are shown in Fig. 10. These were calculated from the time-dependent autocorrelation coefficient defined in a previous paper ${ }^{4}$. Since the autocorrelation functions were asymmetric the rigid lines indicate the spectra based on a positive delay time of the autocorrelation function and the broken lines, those based on a negative delay time. Thus the difference between the two lines indicates the change in energy at any instant. It would appear that the developing wave consisted of a very low-frequency component which increases in magnitude with time, whereas after the propagation instant the higherfrequency components appear so that the spectrum takes on a form found with steady turbulent flows. 

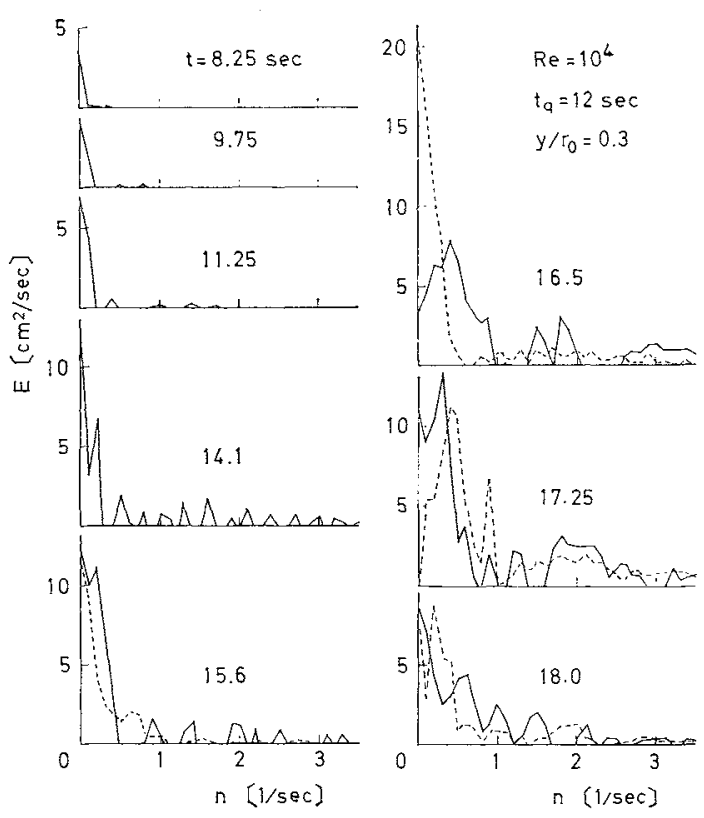

Fig. 10 Energy spectra of $u$-fluctuations at $y / r_{0}=0.3$

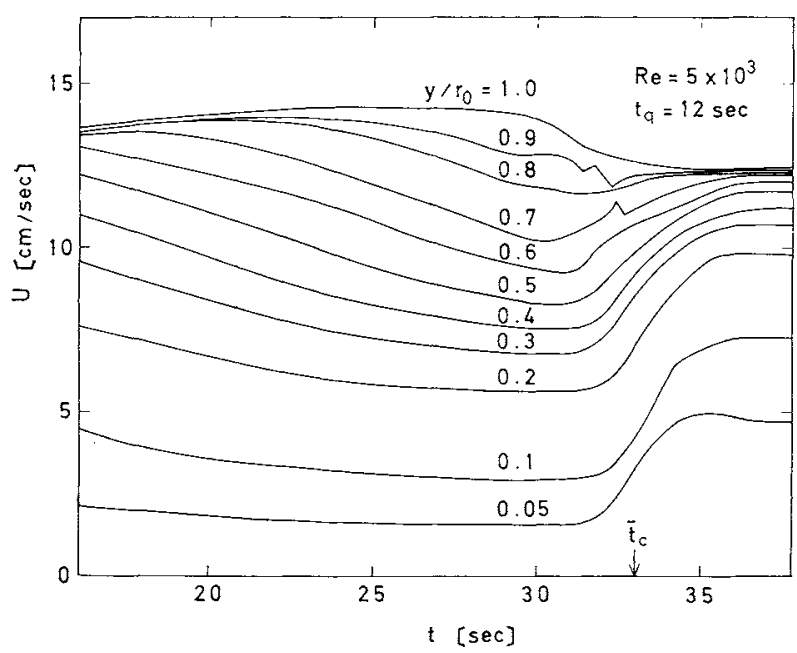

Fig. 11 Variation of ensemble-averaged velocities at various radial positions $\left(R e=5 \times 10^{3}\right)$

In addition, the large difference between the two lines at $t=16.5 \mathrm{sec}$ indicates that the high-frequency components appear suddenly. This abrupt change in the spectrum and a continuous decrease in intensity of $u$ fluctuation strongly suggests that the change at the interface is not a passive one, caused by convection of the flow, but an active one which propagates the interface in the downstream direction. That is, a wave of low frequency and large scale generates and develops continuously in front of the interface. It finally breaks at its critical condition and triggers the downstream propagation of the laminar-turbulent interface.

For transition to turbulence in steady pipe flow, Wygnanski and Champagne ${ }^{5}$ suggested a similar large-eddy structure in the non-turbulent region in front of the interface. However, their measurements are inconsistent with the results of this study since they showed negligibly small intensities in the $u$ fluctuations in the non-turbulent region. This difference, we infer, was caused by their use of a highpass filter in their measurements.

Detailed reasons as to why peaks in the localized radial positions occur are difficult to determine, but some part of the answer must lie with the uniqueness of the position of the peak, that is, the position where the change in average velocity is the smallest at the propagation instant. This was confirmed at other Reynolds numbers. The variations in the ensembleaveraged velocities at $R e=5 \times 10^{3}$ are shown in Fig. 11, and the corresponding profiles of intensity of $u$ fluctuations in Fig. 12. Although the radial position of the peak $\left(y / r_{0} \simeq 0.8\right)$ is nearer the centre of the tube compared with $R e=10^{4}$, it does however coincide with the position of the smallest change in average velocity at $t=\overline{t_{C}}$.

Thus the position of the peak is determined not only by the velocity profile of non-turbulent flow before the transition but also by that of turbulent flow after the transition. This suggests that the development of the wave may be associated with some occurrences at an upstream interface, such as simultaneous rapid acceleration and deceleration in average velocity around the position where the change in velocity is smallest.

A similar spatial structure would also exist in the transition to turbulence in steady-state pipe flow, which differs from starting flow in its non-turbulent velocity profile. In fact, as described before, the timedependent profile of this starting flow would agree with the steady-state profile if the measuring position was sufficiently far downstream where the steady Hagen-Poiseuille profile would be established before the interface propagates there.

\section{3 Propagation into turbulence-decaying flow}

The mechanism of propagation of the interface in decaying turbulence is discussed by using the results of two quieting times $(2.5 \mathrm{sec}, 1.5 \mathrm{sec})$. Variations in the ensemble-averaged velocities are shown in Fig. 13 for quieting time, $1.5 \mathrm{sec}$, and the corresponding profiles of the intensity of $u$-fluctuations in Fig. 14. The latter shows decaying turbulence occurring at the central region during the early part of the transient. At the same time, a peak develops at the characteristic radial position $\left(y / r_{0}=0.1\right)$, where the time change in the average velocity is smallest at the instant of propagation. The results for $t_{q}=2.5 \mathrm{sec}$ were similar showing of the characteristic radial position $\left(y / r_{0}=0.2\right)$.

In these cases, however, the $y / r_{0}$ values are less than those at longer quieting times and seem to decrease with decreasing quieting time. This tendency corresponds to an increase in propagation velocity and consequently to a decrease in time for development of 

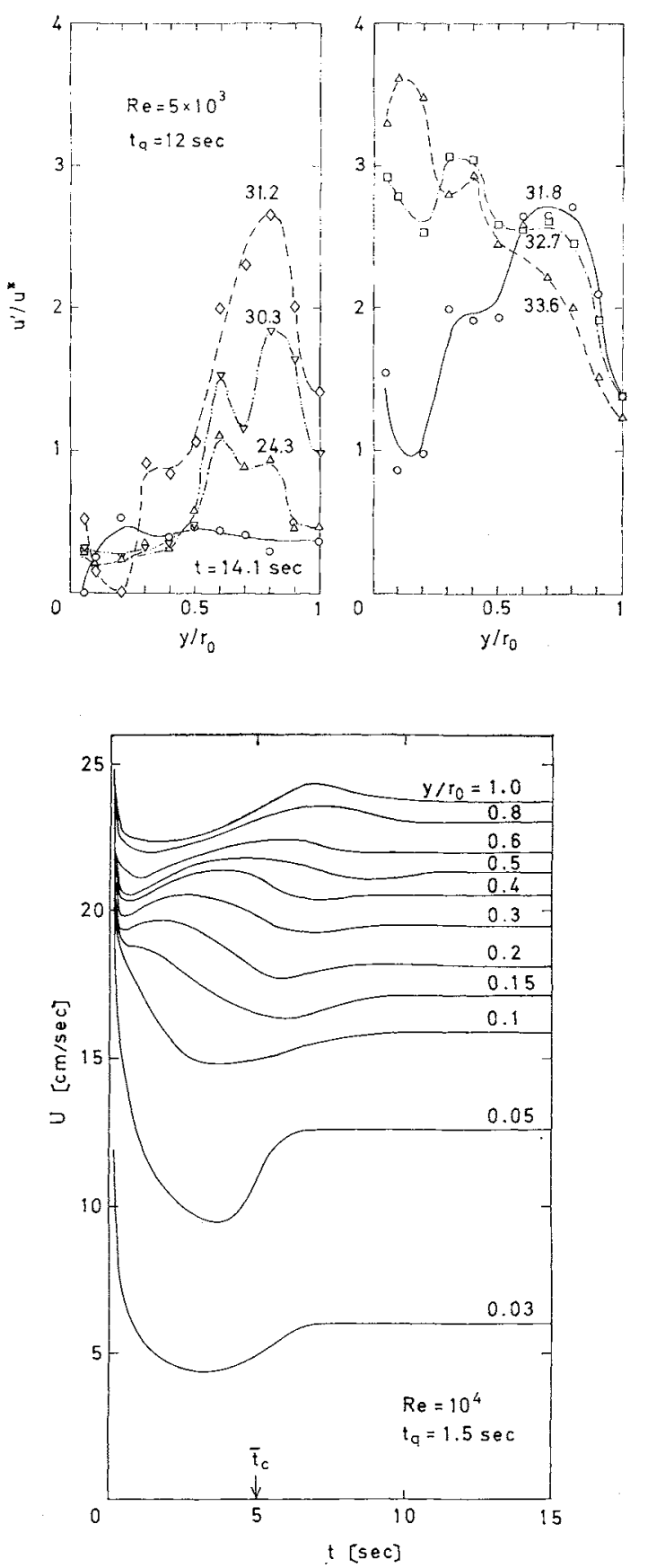

Fig. 13 Variation of ensemble-averaged velocities in starting flow after a short quieting time $\left(t_{q}=1.5\right.$ sec)

the decelerating region which will then be accelerated at the propagation of the interface.

Let us consider the mechanism of the increase in propagation velocity on the basis of some peculiar features observed about propagation time, such as its strong dependence on separation distance between electrode pairs alinged to the flow direction and its large deviation from the mean value. The dependence of propagation velocity on longitudinal separation distance $\Delta x$ is shown in Fig. 15. It is noted that at small separation, the propagation velocity is nearly

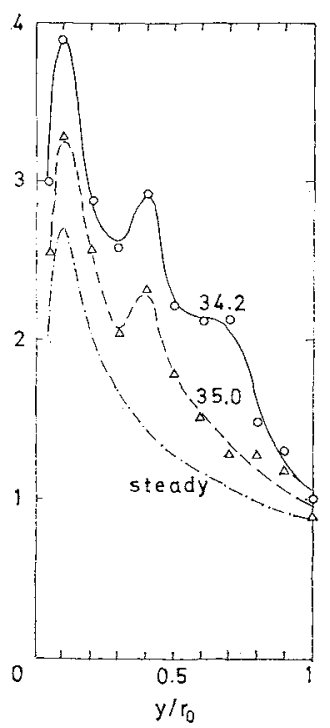

Fig. 12 Profiles of intensity of $u$-fluctuations $\left(\operatorname{Re}=5 \times 10^{3}\right)$

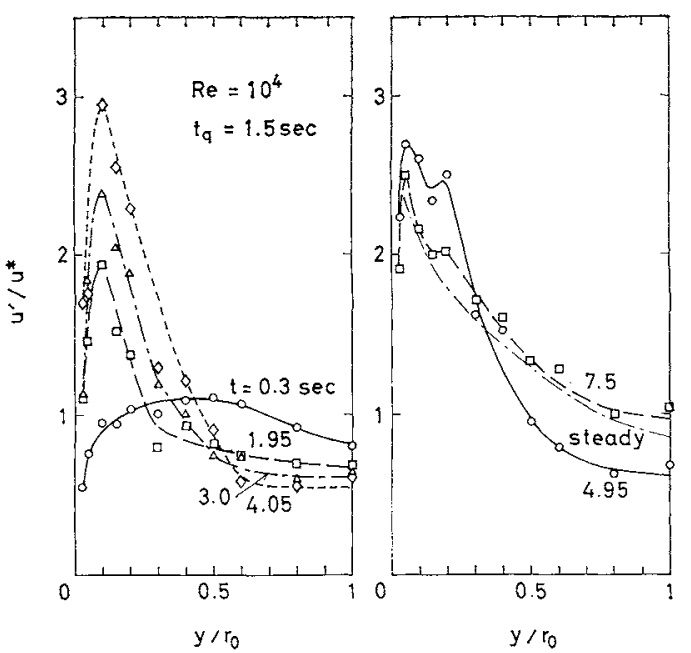

Fig. 14 Profiles of intensity of $u$-fluctuations in starting flow after a short quieting time $\left(t_{q}=1.5 \mathrm{sec}\right)$

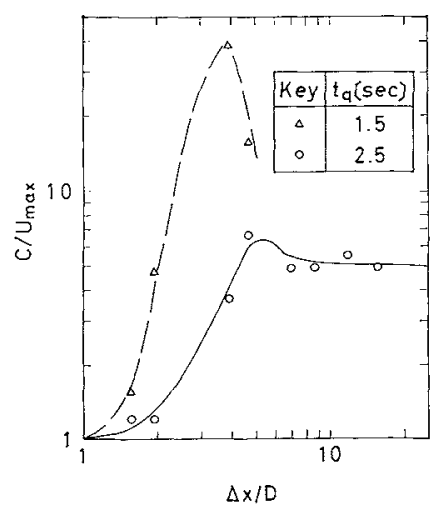

Fig. 15 Propagation velocity as a function of longitudinal separation

equal to $U_{\max }$, the same velocity as the propagation in turbulence-decayed flow. Note also that the velocity becomes very large at separations of $3 D-6 D$ (it should 


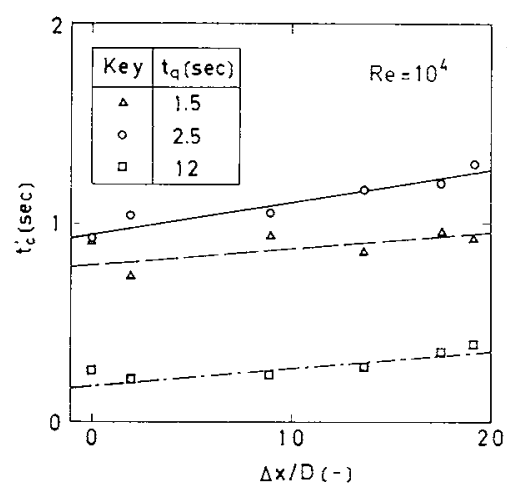

Fig. 16 Standard deviation of the propagation time

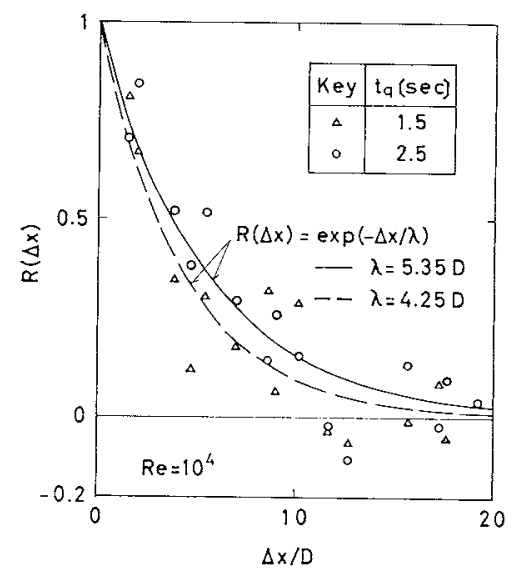

Fig. 17 Longitudinal correlations of $t_{C}$-fluctuations

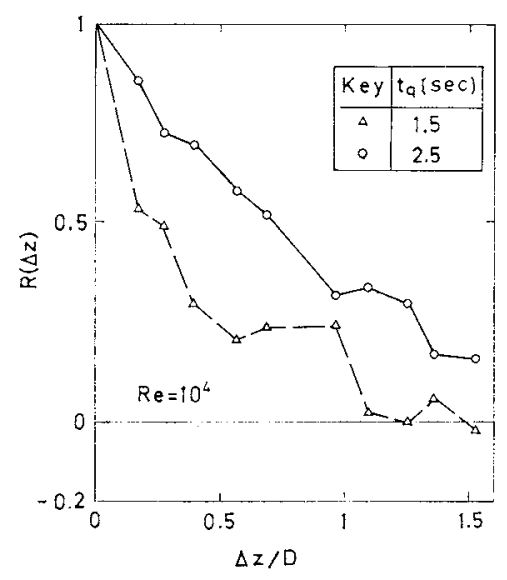

Fig. 18 Circumferential correlations of $\boldsymbol{t}_{C}$-fluctuation

be remembered that the velocity is a mean value integrated from zero to the separation distance). A re-examination of the data records showed that an apparent upstream propagation was sometimes included in the measurements at the latter separations.

The standard deviation $t_{C}^{\prime}$ and the space correlations, $R(\Delta x)$ and $R(\Delta z)$, of the propagation time are shown in Figs. 16, 17 and 18. These are defined as:

$$
\begin{gathered}
t_{\sigma}^{\prime}(x)=\sqrt{\left\{t_{\sigma}(x)-t_{C}(x)\right\}^{2}} \\
R(\Delta x)=\frac{\sqrt\left[\{ t _ { \sigma } ( x ) - t _ { C } ( x ) ] { t ^ { 2 } } \left\{t_{\sigma}(x+\Delta x)-\overline{\left.t_{\sigma}(x+\Delta x)\right\}}\right.\right.}{t_{\sigma}^{\prime}(x) t_{\sigma}^{\prime}(x+\Delta x)}
\end{gathered}
$$

The functional form of $R(\Delta z)$ is the same as Eq. (2). Here the overlines signify the ensemble averages.

The results show that the deviation $t_{C}^{\prime}$ in Fig. 16 has a longitudinal scale which is one order of magnitude larger than the circumferential scale. Also shown in Fig. 17 is the correlation function:

$$
R(\Delta x)=\exp (-\Delta x / \lambda)
$$

It represents the correlation coefficient of the random rectangular wave of a constant amplitude with a mean wave length $\lambda$. It should be pointed out that both values $(4.25 D, 5.35 D)$ of the mean wave length obtained by the correlation of the results are comparable to the separations for the maximun propagation velocity.

The above-clarified facts suggest that the rapid propagation is not continuous but is caused by a random jump which is known in the transition to turbulence in steady pipe flow under highly disturbed inlet conditions $^{2}$. The jump makes another interface in front of the former one, enclosing a non-turbulent region between them. It occurs randomly in space and time, but has such a preferred longitudinal scale that it appeared as the limiting range of the separation for the maximum propagation velocity. Furthermore, if the enclosed non-turbulent region is assumed to be consumed by the turbulent region with velocity $U_{\max }$, the deviation of the propagation time can be related to the wave length by $t_{C}^{\prime} \sim \lambda / U_{\max }$. The two values of $\lambda / U_{\max }, 0.916 \mathrm{sec}$ and $1.153 \mathrm{sec}$, are comparable to $t_{C}^{\prime}$. Thus the large deviation of propagation time is attributable to the longitudinal scale of the jump.

However, the difference in the longitudinal scale of the jump between the two quieting times is too small to explain the difference in propagation velocity. Therefore it is not the scale but rather the frequency of occurrence of the jump that increases the propagation velocity with decreasing quieting times. For the circumferential scale, Fig. 18 shows a decreasing tendency with decreasing quieting time, so that the jump would be localized randomly in the circumferential direction in addition to the wallward approach of the unstable wave. This tendency implies that propagation in turbulence-decaying flow continuously alters to the wall streaky structure in steady turbulent flow with decreasing quieting time. Unfortunately, experimental verification of this inference was prevented by a difficulty in discriminating propagating turbulence from the high level of decaying turbulence in measurements with quieting times smaller than $1.5 \mathrm{sec}$. 


\section{Conclusion}

The transition to turbulence in pipe flow started impulsively with a supercritical Reynolds number propagates downstream with a precise interface by consuming the non-turbulent region where laminar boundary-layer flow is developing with time.

In the process of interface propagation, a wave of very low frequency is generated in the non-turbulent region and develops centring around the radial position where the time change in velocity is smallest at the instant of propagation. The wave finally breaks and this continuously triggers the downstream propagation of the turbulent-flow region at a constant velocity equal to the maximum velocity of the steadystate turbulent flow created by the transition to turbulence.

In addition, the jump of the turbulent-non-turbulent interface occurs in the propagation in turbulencedecaying flow. The propagation velocity becomes much larger than $U_{\max }$ and increases with decreasing quieting time between shutdown and restart.

\section{Acknowledgments}

This research was supported by the Scientific Research Funds of the Ministry of Education, Science and Culture, Japan under Grant No. 275096. In addition, the first author obtained financial support from the Sakkokai Foundation.

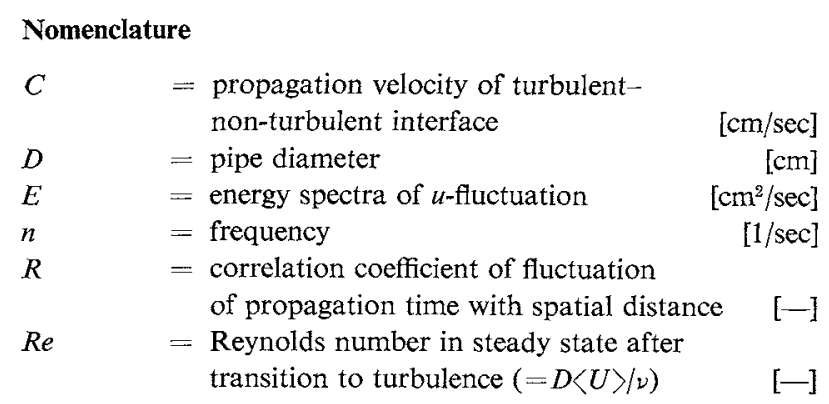

\begin{tabular}{|c|c|c|}
\hline$r_{0}$ & $=$ radius of circular tube & [cm] \\
\hline$t$ & $=$ time from startup of flow & [sec] \\
\hline$t_{C}$ & $=$ propagation time (see Fig. 2 ) & [sec] \\
\hline$\overline{t_{C}}$ & $=$ mean value of $t_{C}$ & [sec] \\
\hline$t_{C}^{\prime}$ & $=$ standard deviation of propagation time & [sec] \\
\hline$t_{q}$ & $=$ quieting time from shutdown to restart & [sec] \\
\hline$U$ & $\begin{array}{l}=\text { statistically averaged velocity in axial } \\
\text { direction }\end{array}$ & {$[\mathrm{cm} / \mathrm{sec}]$} \\
\hline$U_{\max }$ & $=$ maximum velocity in turbulent flow & {$[\mathrm{cm} / \mathrm{sec}]$} \\
\hline$\langle U\rangle$ & $\begin{array}{l}=\text { velocity obtained by averaging } U \\
\text { over the cross-section }\end{array}$ & {$[\mathrm{cm} / \mathrm{sec}]$} \\
\hline$u$ & $\begin{array}{l}=\text { fluctuating component of velocity in } \\
\text { axial direction }\end{array}$ & {$[\mathrm{cm} / \mathrm{sec}]$} \\
\hline$u^{\prime}$ & $=$ root-mean square of $u$ & {$[\mathrm{~cm} / \mathrm{sec}]$} \\
\hline$u^{*}$ & $\begin{array}{l}=\text { friction velocity in steady state after } \\
\text { transition to turbulence }\left(=\sqrt{\tau_{w} / \rho}\right)\end{array}$ & {$[\mathrm{cm} / \mathrm{sec}]$} \\
\hline$x$ & $=$ distance in longitudinal direction & [cm] \\
\hline$\Delta x$ & $=$ separation distance in longitudinal dire & ction $[\mathrm{cm}]$ \\
\hline$y$ & $=$ normal distance from wall & {$[\mathrm{cm}]$} \\
\hline$\Delta z$ & $\begin{array}{l}=\text { separation distance in circumferential } \\
\text { direction }\end{array}$ & {$[\mathrm{cm}]$} \\
\hline$\lambda$ & $=$ wave length & {$[\mathrm{cm}]$} \\
\hline$\mu$ & $=$ viscosity & $\mathrm{g} / \mathrm{cm} \cdot \mathrm{sec}]$ \\
\hline$\nu$ & $=$ kinematic viscosity & {$\left[\mathrm{cm}^{2} / \mathrm{sec}\right]$} \\
\hline$\rho$ & $=$ density & {$\left[\mathrm{g} / \mathrm{cm}^{3}\right]$} \\
\hline$\tau_{w}$ & $=$ shear stress at wall & $\left./ \mathrm{cm} \cdot \mathrm{sec}^{2}\right]$ \\
\hline
\end{tabular}

\section{Literature Cited}

1) Klebanoff, P.S., K. D. Tidstrom and L. M. Sargent: J. Fluid Mech., 12, 1 (1962).

2) Lindgren, E. R.: Phys. Fluids, 12, 418 (1969).

3) Maruyama, T., T. Kuribayashi and T. Mizushina: $J$. Chem. Eng. Japan, 9, 431 (1976).

4) Maruyama, T., K. Fujii and T. Mizushina: ibid., 10, 421 (1977).

5) Wygnanski, I. J. and F. H. Champagne: J. Fluid Mech., 59, 281 (1973).

(Presented in part at the 11th Autumn Meeting of The Soc. of Chem. Engrs., Japan at Tokyo 1977.) 